\title{
Is There a Role for Colchicine in Acute Coronary Syndromes? A Literature Review
}

\author{
Jahanzeb Malik $^{1}$, Nismat Javed ${ }^{2,1}$, Uzma Ishaq ${ }^{3}$, Umar Khan ${ }^{4}$, Talha Laique ${ }^{5}$ \\ 1. Cardiology, Rawalpindi Institute of Cardiology, Rawalpindi, PAK 2. Internal Medicine, Shifa College of Medicine - \\ Shifa Tameer-E-Millat University, Islamabad, PAK 3. Hematology and Medical Oncology, Fauji Foundation Hospital, \\ Rawalpindi, PAK 4. Pulmonary Medicine, University Hospital Limerick, Limerick, IRL 5. Pharmacology, Lahore Medical \\ and Dental College, Lahore, PAK
}

Corresponding author: Jahanzeb Malik, heartdoc86@gmail.com

\begin{abstract}
Inflammation is identified as a keystone of atherosclerosis. This review of the literature explores the unique anti-inflammatory effects of colchicine and summarizes the mechanisms of inflammation in acute coronary syndrome. It outlines other therapeutic strategies employed until now to target coronary inflammation and analyzes the role of colchicine in improving the outcomes of acute coronary syndrome. Despite the existence of guideline-directed medical therapy, there still remains a higher risk for recurrence due to continuous inflammation at remaining vascular sites. Several anti-inflammatory strategies have been employed, but they have not been shown to be beneficial. However, colchicine is becoming increasingly popular in tackling this problem. For this review, databases were searched for trials on the role of colchicine as an anti-inflammatory therapy in acute coronary syndrome.
\end{abstract}

Categories: Cardiology, Internal Medicine, Miscellaneous

Keywords: acute coronary syndrome, colchicine, atherosclerosis, ischemic heart disease, inflammation

\section{Introduction And Background}

Atherosclerosis is the leading cause of morbidity and mortality worldwide, mostly due to coronary artery disease causing acute coronary syndrome. Guideline-directed medical therapy puts emphasis on the modulation of cardiovascular risk factors like control of diabetes, lipid-lowering therapies, and antiplatelet medications to prevent plaque rupture and thrombus formation. However, increasing evidence is now available that implicates inflammation as the core process for the pathogenesis of atherosclerotic plaque, its changing dynamics, and rupture $[1,2]$. Patients with a known history of acute coronary syndrome are at an elevated risk for recurrent major adverse cardiovascular events [3]. This is mainly due to the residual inflammatory process in coronary arteries [4].

Received 05/01/2020 Review began 05/09/2020 Review ended 05/10/2020 Published 05/17/2020

(c) Copyright 2020 Malik et al. This is an open access article distributed under the terms of the Creative Commons Attribution License CC-BY 4.0., which permits unrestricted use, distribution, and reproduction in any medium, provided the original author and source are credited.
Statins, used in guideline-directed medical therapy to lower target low-density lipoprotein cholesterol (LDL-C), have been known to possess an anti-inflammatory function in addition to their lipid-lowering effects [5]. Treatment, however, is now shifting towards specific anti-inflammatory approaches to further improve outcomes in these patients. Recent trials have laid emphasis on the hypothesis that highlights the role of inflammation in atherothrombosis. The Canakinumab Anti-inflammatory Thrombosis Outcome Study (CANTOS) found that canakinumab at a dose of $150 \mathrm{mg}$ every three months reduced adverse cardiovascular events in patients with previous myocardial infarction and C-reactive protein (CRP) levels of

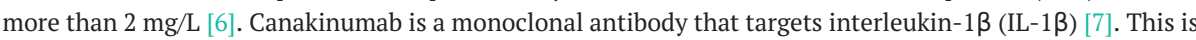
an important inflammatory cascade in the interleukin-6 (IL-6) signaling pathway. However, this is not costeffective and it causes high rates of fatal infections, thus limiting its widespread use.

Colchicine, on the other hand, is a widely available, inexpensive, and tolerable anti-inflammatory medication. It prevents mitosis by inhibiting microtubule polymerization $[3,8]$. It is mainly used in the management of gout, pericarditis, and familial Mediterranean fever. This review outlines the mechanism of inflammation in acute coronary syndrome and gives an overview of some targeted anti-inflammatory approaches, leading to the evidence that endorses colchicine as an agent for secondary prevention after acute coronary syndrome.

\section{Review}

\section{Methods}

To summarize the body of available evidence, a scoping review design was used to incorporate a range of studies and reports in a narrative format. For this purpose, we identified the relevant literature by performing a search of the bibliographic databases of MEDLINE and ClinicalTrials using the keywords "colchicine", "atherosclerosis", and "colchicine" and "acute coronary syndrome". We reviewed the literature for the previous 20 years. 


\section{The NOD-like receptor protein 3 (NLRP3) inflammasome and acute coronary syndrome}

There is a lot of evidence that local and systemic inflammation plays a pathologic role in acute coronary syndrome. Understanding these mechanisms can lead to newer therapeutic agents and strategies $[3,9]$. By certain triggers like LDL-C, endothelial activation occurs, releasing pro-inflammatory cytokines, metalloproteinases, and oxygen-free radicals, which increase inflammation and possibly reduce plaque stability by weakening the fibrous cap. Further recruitment of pro-inflammatory cytokines causes inflammatory cell infiltration, which potentiates the pro-coagulant properties of these cells. Rupture of the fibrous cap causes contact of these inflammasomes with blood, leading to thrombosis [9-12].

A specific kind of neutrophils is recognized as an important atheroinflammation contributor causing plaque rupture [13]. Another key player in the innate immune system is the NLRP3 inflammasome. It is a multiprotein compound present in myeloid cells, including neutrophils and eosinophils. Exposure to stress signals is sensed by an NLRP3 receptor, which leads to the assembly of NLRP3 as an adaptor protein apoptosis-associated speck-like substance that contains the caspase recruitment domain [3,14]. Adenosine triphosphate (ATP) activates caspase-1, leading to the secretion of active IL-1 $\beta$ and IL-18. Exposure of neutrophils to stimuli like cholesterol crystals causes activation of NLRP3 inflammasome. This, in turn, promotes the caspase-1-dependent release of two key inflammatory cytokines (IL-1 $\beta$ and IL-18), both of which are predictive of future cardiovascular events and are key mediators in plaque development and destabilization.

IL- $1 \beta$ is a proinflammatory cytokine that causes adhesion of monocytes to vascular surfaces. It induces procoagulant activity via the growth of vascular smooth muscle cells [15]. The activation of IL- $1 \beta$ signals IL6 , which drives the expression of atherothrombosis mediators. It has been found that patients with acute coronary syndrome have the highest transcoronary numbers of IL-1 $\beta$, IL-6, and IL-18 compared to those with chronic coronary syndromes [15].

This shows that a transformation of atherosclerotic plaque from stable to rupture is mediated by inflammatory cells that destabilize the lesion causing thrombosis and myocardial infarction. However, some studies show that there is widespread activation of neutrophils across the coronary bed in unstable angina, regardless of the culprit lesion [16].

\section{Various agents used for targeting inflammation in acute coronary syndromes}

The role of inflammation in acute coronary syndrome has led to several novel therapeutic anti-inflammatory agents being brought to decrease recurrent events under trials. However, despite many promising phase II trials, many of these have not shown any clinical superiority than guideline-directed medical therapy [17].

Statins have pleiotropic anti-inflammatory properties acting synergistically with lipid-lowering. Their mechanism of action includes T-cell activation, leukocyte adhesion, and enhancing endothelial nitric oxide production [5]. Several large trials have shown that statins decrease CRP along with their lipid-lowering effects. The Pravastatin or Atorvastatin Evaluation and Infection Therapy (PROVE-IT) trial enrolled 3,745 patients with acute coronary syndrome to undergo moderate or high-dose statin therapy and concluded that patients with lower CRP had lower cardiovascular events [9]. Similarly, the Improved Reduction of Outcomes; Vytorin Efficacy International Trial (IMPROVE-IT) randomized 18,144 patients after stabilized acute coronary syndrome to simvastatin and measured CRP and LDL-C. Similar to the PROVE-IT trial, there was an almost identical proportion of patients who met the primary composite endpoint (cardiovascular death, acute coronary syndrome, coronary revascularization within 30 days, or nonfatal stroke) in the group that achieved CRP levels of $<2 \mathrm{mg} / \mathrm{L}$ but LDL-C levels of $>1.8 \mathrm{mmol} / \mathrm{L}[9,18]$.

Corticosteroids were also used as agents for anti-inflammatory response in acute coronary syndrome. A meta-analysis of 11 trials showed a $26 \%$ decrease in mortality; however, the data was insufficient, and there arose a risk of cardiac rupture secondary to impaired wall healing [19]. Apart from aspirin, nonsteroidal antiinflammatory drugs were found to elevate the risk of cardiovascular events [20].

Certain monoclonal antibodies like inclacumab and pexelizumab were tested but no difference in mortality and morbidity was found [21]. However, one monoclonal antibody that targeted IL-1 $\beta$, canakinumab, has gained attention recently. Canakinumab Anti-inflammatory Thrombosis Outcome Study (CANTOS) was a randomized trial that evaluated canakinumab along with guideline-directed medical therapy to prevent recurrent cardiovascular events [6]. 10,061 patients with myocardial infarction were included in the study. At 48 months, there was a reduction of CRP at $26 \%$ from $50 \mathrm{mg}$ dose and $37 \%$ from $150 \mathrm{mg}$ dose. At a follow-up at almost four years, the cardiovascular event was found to be reduced by $15 \%$ in the $150-\mathrm{mg}$ group compared to the placebo $(\mathrm{P}=0.021)$. There was no difference in all-cause mortality.

\section{Role of colchicine}


Colchicine is a widely available, inexpensive anti-inflammatory drug with well-known side effects. Common adverse effects are nausea, vomiting, and diarrhea. Rare side effects include hepatic injury, rhabdomyolysis, hypersensitivity, and blood disorders. Major interactions are with statins and cytochrome P450 3A4 inhibitors [3,22-27]. It acts by microtubule depolymerization and tubulin binding, inhibiting the NLRP3 inflammasome protein complex [28,29]. By monocyte caspase-1 inhibition, it brings about inhibition of colocalization of inflammasome cytoplasmic proteins and inhibition of $\mathrm{P} 2 * 7$-mediated pore function, a key step in the NLRP3 response to adenosine triphosphate [30-32]. In patients with acute coronary syndrome, the monocytes exhibited a reduction in secreted levels of IL-1 $\beta$ after they received a low dose of colchicine. Reduction in procaspase- 1 was also seen along with trans-coronary caspase-1-mediated cytokines (IL-1 $\beta$ and IL-18) by $88 \%$ [33]. These findings confirm that local cholesterol crystal activation causes inflammation within the atherosclerotic plaque, leading to plaque instability and rupture [7,34].

The Low-Dose Colchicine (LoDoCo) trial, which consisted of 532 patients with chronic coronary syndrome who were followed up for three years, showed that colchicine $0.5 \mathrm{mg}$ per day administered along with guideline-directed medical therapy was beneficial in the prevention of adverse cardiovascular events $(\mathrm{P}=0.001)$ [35]. In an observational study involving 80 patients with acute coronary syndrome, patients received either guideline-directed medical therapy alone or guideline-directed medical therapy along with colchicine $0.5 \mathrm{mg}$ per day for a year. CT coronary angiography performed at 12 months showed plaque stabilization and change in total atheroma volume. There was a change in low attenuation plaque (LAP) volume with a mean reduction of $41 \%$ in the treatment group and $17 \%$ in the control group $(\mathrm{P}=0.008)$. Similarly, there was a reduction of CRP levels in both groups (37\% and $15 \%$ in the treatment and control group, respectively) $(\mathrm{P}=0.001)$. This study demonstrated that colchicine combined with guideline-directed medical therapy has a more plaque stabilizing effect than guideline-directed medical therapy alone [36].

In an observational study involving 80 patients who had undergone acute coronary syndrome and ischemic stroke, no difference in CRP was found in treatment versus the control group after administering colchicine and following up for 30 days [37]. On the contrary, a study of 64 patients found that low-dose colchicine reduced CRP levels in patients with chronic coronary syndrome [35].

The landmark Colchicine Cardiovascular Outcomes Trial (COLCOT) was a metacentric trial that randomized 4,745 patients with either low-dose colchicine ( $0.5 \mathrm{mg}$ per day) or placebo. Among patients with recent myocardial infarction on guideline-directed medical therapy, $0.5 \mathrm{mg}$ of colchicine daily reduced the risk of cardiovascular death, acute myocardial infarction, stroke, resuscitated cardiac arrest, or urgent hospitalization for angina requiring revascularization by $23 \%$. Overall rates of adverse events were low with colchicine ( $0.9 \%$ vs $0.4 \% ; \mathrm{P}=0.03$ ). This clearly showed the benefit of adding colchicine to guideline-directed medical therapy in patients with acute coronary syndrome [38].

\section{Conclusions}

With the knowledge that inflammation plays a pathologic role in atherosclerosis, new strategies for the treatment and prevention of coronary artery disease can be established. Many anti-inflammatory therapies have been tested during the past decade; however, they have failed to show major differences in outcomes when tested in randomized trials. The role of therapies, particularly colchicine, will hopefully be defined well in future studies.

\section{Additional Information \\ Disclosures}

Conflicts of interest: In compliance with the ICMJE uniform disclosure form, all authors declare the following: Payment/services info: All authors have declared that no financial support was received from any organization for the submitted work. Financial relationships: All authors have declared that they have no financial relationships at present or within the previous three years with any organizations that might have an interest in the submitted work. Other relationships: All authors have declared that there are no other relationships or activities that could appear to have influenced the submitted work.

\section{References}

1. Geovanini GR, Libby P: Atherosclerosis and inflammation: overview and updates . Clin Sci (Lond). 2018, 132:1243-1252. 10.1042/CS20180306

2. Pant S, Deshmukh A, Gurumurthy GS, Pothineni NV, Watts TE, Romeo F, Mehta JL: Inflammation and atherosclerosis--revisited. J Cardiovasc Pharmacol Ther. 2014, 19:170-178. 10.1177/1074248413504994

3. Martinez GJ, Celermajer DS, Patel S: The NLRP3 inflammasome and the emerging role of colchicine to inhibit atherosclerosis-associated inflammation. Atherosclerosis. 2018, 269:262-271. 10.1016/j.atherosclerosis.2017.12.027

4. Stone GW, Maehara A, Lansky AJ, et al.: A prospective natural-history study of coronary atherosclerosis . N Engl J Med. 2011, 364:226-235. 10.1056/NEJMoa1002358

5. Liberale L, Carbone F, Montecucco F, Sahebkar A: Statins reduce vascular inflammation in atherogenesis: a review of underlying molecular mechanisms. Int J Biochem Cell Biol. 2020, 122:105735.

10.1016/j.biocel.2020.105735 
6. Ridker PM, Everett BM, Thuren T, et al.: Antiinflammatory therapy with canakinumab for atherosclerotic disease. N Engl J Med. 2017, 377:1119-1131. 10.1056/NEJMoa1707914

7. Vaidya K, Martínez G, Patel S: The role of colchicine in acute coronary syndromes . Clin Ther. 2019, 41:1120. 10.1016/j.clinthera.2018.07.023

8. Shah B, Pillinger M, Zhong $\mathrm{H}$, et al.: Effects of acute colchicine administration prior to percutaneous coronary intervention: COLCHICINE-PCI randomized trial. Circ Cardiovasc Interv. 2020, $13: \mathrm{e} 008717$. 10.1161/CIRCINTERVENTIONS.119.008717

9. Ridker PM, Cannon CP, Morrow D, et al.: C-reactive protein levels and outcomes after statin therapy . N Engl J Med. 2005, 352:20-28. 10.1056/NEJMoa042378

10. Ridker PM, Narula J: Will reducing inflammation reduce vascular event rates?. JACC Cardiovasc Imaging. 2018, 11:317-319. 10.1016/j.jcmg.2017.10.001

11. Mulvihill NT, Foley JB: Inflammation in acute coronary syndromes. Heart. 2002, 87:201-204. 10.1136/heart.87.3.201

12. Nanchen D, Klingenberg R, Gencer B, et al.: Inflammation during acute coronary syndromes - risk of cardiovascular events and bleeding. Int J Cardiol. 2019, 287:13-18. 10.1016/j.ijcard.2019.03.049

13. Martines GJ, Barraclough JY, Nakhla S, et al.: Neutrophil-derived microparticles are released into the coronary circulation following percutaneous coronary intervention in acute coronary syndrome patients. Biosci Rep. 2017, 37:BSR20160430. 10.1042/BSR20160430

14. Gonzalez-Pacheco H, Vargas-Alarcon G, Angeles-Martinez J, et al.: The NLRP3 and CASP1 gene polymorphisms are associated with developing of acute coronary syndrome: a case-control study. Immunol Res. 2017, 65:862-868. 10.1007/s12026-017-8924-0

15. Kang HJ, Bae KY, Kim SW, et al.: Relationship between interleukin-1 $\beta$ and depressive disorder after acute coronary syndrome. Prog Neuropsychopharmacol Biol Psychiatry. 2017, 72:55-59. 10.1016/j.pnpbp.2016.09.001

16. Odeberg J, Freitag M, Forssell H, et al.: Influence of pre-existing inflammation on the outcome of acute coronary syndrome: a cross-sectional study. BMJ Open. 2016, 6:e009968. 10.1136/bmjopen-2015-009968

17. Fernandes JL, de Oliveira RT, Mamoni RL, Coelho OR, Nicolau JC, Blotta MH, Serrano CV Jr: Pentoxifylline reduces pro-inflammatory and increases anti-inflammatory activity in patients with coronary artery disease--a randomized placebo-controlled study. Atherosclerosis. 2008, 196:434-442. 10.1016/j.atherosclerosis.2006.11.032

18. Bohula EA, Giugliano RP, Cannon CP, et al.: Achievement of dual low-density lipoprotein cholesterol and high-sensitivity C-reactive protein targets more frequent with the addition of ezetimibe to simvastatin and associated with better outcomes in IMPROVE-IT. Circulation. 2015, 132:1224-1233. 10.1161/CIRCULATIONAHA.115.018381

19. Vogel RA, Forrester JS: Cooling off hot hearts: a specific therapy for vulnerable plaque? . J Am Coll Cardiol. 2013, 61:411-412. 10.1016/j.jacc.2012.10.026

20. Bueno H, Bardají A, Patrignani P, Martín-Merino E, García-Rodríguez LA: Use of non-steroidal antiinflammatory drugs and type-specific risk of acute coronary syndrome. Am J Cardiol. 2010, 15:11021106. 10.1016/j.amjcard.2009.12.008

21. Stähli BE, Gebhard C, Duchatelle V, et al.: Effects of the P-selectin antagonist inclacumab on myocardial damage after percutaneous coronary intervention according to timing of infusion: insights from the SELECT-ACS trial. J Am Heart Assoc. 2016, 5:e004255. 10.1161/JAHA.116.004255

22. Tabbalat RA, Hamad NM, Alhaddad IA, Hammoudeh A, Akasheh BF, Khader Y: Effect of colchicine on the incidence of atrial fibrillation in open heart surgery patients: END-AF trial. Am Heart J. 2016, 178:102-107. 10.1016/j.ahj.2016.05.006

23. Deftereos S, Giannopoulos G, Papoutsidakis N, et al.: Colchicine and the heart: pushing the envelope. J Am Coll Cardiol. 2013, 62:1817-1825. 10.1016/j.jacc.2013.08.726

24. Frommeyer G, Krawczyk J, Dechering DG, Kochhäuser S, Leitz P, Fehr M, Eckardt L: Colchicine increases ventricular vulnerability in an experimental whole-heart model. Basic Clin Pharmacol Toxicol. 2017, 120:505-508. 10.1111/bcpt.12702

25. Bayes-Genis A, Adler Y, de Luna AB, Imazio M: Colchicine in pericarditis. Eur Heart J. 2017, 38:1706-1709. 10.1093/eurheartj/ehx246

26. Hemkens LG, Ewald H, Gloy VL, et al.: Colchicine for prevention of cardiovascular events . Cochrane Database Syst Rev. 2016, 2016:CD011047. 10.1002/14651858.CD011047.pub2

27. Lazaros G, Imazio M, Brucato A, Vlachopoulos C, Lazarou E, Vassilopoulos D, Tousoulis D: The role of colchicine in pericardial syndromes. Curr Pharm Des. 2018, 24:702-709. 10.2174/1381612824666180116101823

28. Angelidis C, Kotsialou Z, Kossyvakis C, et al.: Colchicine pharmacokinetics and mechanism of action. Curr Pharm Des. 2018, 24:659-663. 10.2174/1381612824666180123110042

29. Yue H, Liang W, Gu J, et al.: Comparative transcriptome analysis to elucidate the therapeutic mechanism of colchicine against atrial fibrillation. Biomed Pharmacother. 2019, 119:109422. 10.1016/j.biopha.2019.109422

30. Liantinioti G, Argyris AA, Protogerou AD, Vlachoyiannopoulos P: The role of colchicine in the treatment of autoinflammatory diseases. Curr Pharm Des. 2018, 24:690-694. 10.2174/1381612824666180116095658

31. Shah SR, Alweis R, Shah SA, et al.: Effects of colchicine on pericardial diseases: a review of the literature and current evidence. J Community Hosp Intern Med Perspect. 2016, 6:31957. 10.3402/jchimp.v6.31957

32. Slobodnick A, Shah B, Krasnokutsky S, Pillinger MH: Update on colchicine, 2017. Rheumatology (Oxford). 2018, 57 :i4-i11. 10.1093/rheumatology/kex453

33. Martinez GJ, Robertson S, Barraclough J, et al.: Colchicine acutely suppresses local cardiac production of inflammatory cytokines in patients with an acute coronary syndrome. J Am Heart Assoc. 2015, 4:e002128. 10.1161/JAHA.115.002128

34. Nidorf SM, Verma S: Is there a role for colchicine in acute coronary syndromes? . J Am Heart Assoc. 2015, 4:e002372. 10.1161/JAHA.115.002372

35. Nidorf SM, Eikelboom JW, Budgeon CA, Thompson PL: Low-dose colchicine for secondary prevention of 


\section{Cureus}

cardiovascular disease. J Am Coll Cardiol. 2013, 61:404-410. 10.1016/j.jacc.2012.10.027

36. Vaidya K, Arnott C, Martínez GJ, et al.: Colchicine therapy and plaque stabilization in patients with acute coronary syndrome: a CT coronary angiography study. JACC Cardiovasc Imaging. 2018, 11:305-316.

10.1016/j.jcmg.2017.08.013

37. Raju NC, Yi Q, Nidorf M, Fagel ND, Hiralal R, Eikelboom JW: Effect of colchicine compared with placebo on high sensitivity C-reactive protein in patients with acute coronary syndrome or acute stroke: a pilot randomized controlled trial. J Thromb Thrombolysis. 2012, 33:88-94. 10.1007/s11239-011-0637-y

38. Tardif JC, Kouz S, Waters DD, et al.: Efficacy and safety of low-dose colchicine after myocardial infarction . N Engl J Med. 2019, 26:2497-2505. 10.1056/NEJMoa1912388 